\title{
Influence of Crosswind on Aerodynamic Performance of Wind Turbine in Wind-drive Device
}

\author{
Si-Yu WANG, Hong-Bin LI, Ye-Kun ZHEN, Xiao-Qian XU, Tao-Hui SHI \\ Faculty of Mechanical Engineering and Mechanics, Ningbo University, Ningbo 315211, China
}

1358535992@qq.com

Keywords: Crosswind, Velocity Decomposition, Aerodynamic Performance, Local Impact.

\begin{abstract}
To enhance the utilization rate of wind energy, introduce a new kind of Movable Wind-drive Device and research the influence of crosswind on aerodynamic performance of wind turbine in wind-drive device. Based on blade coordinate system and feline coordinate system, carry on a vector decomposition of wind velocity and obtain the change rules of every velocity component. According to the classical momentum-blade theory and strip analysis theory, analyze the aerodynamic model in positive direction wind and in negative direction wind, research the difference of the formulas in two cases, get the impact of the crosswind on wind turbine blades. Calculation results express that axial thrust and torque possess periodic local impact on the middle section of wind turbine.
\end{abstract}

\section{Foreword}

In order to improve the utilization rate of wind energy, wind speed increases with the increase of wind power generation system using, put forward a contraction tube can move along the track of the wind collecting device, focuses on the study of crosswind on device of wind turbine aerodynamic effect. Crosswind factors will lead to wind turbine blade vibration, greatly increasing the early damage of each component of the wind turbine [1-3]. Wind the wind load distribution is not uniform, is the main reason causing blade wielding [4-5]. Zheng Liming put the wind velocity is decomposed into mean and fluctuation components of components to consider the dynamic response of wind turbine [6]. Wu Yan [7] analysis of wind turbine wind gas partial dynamic characteristics calculation, get the side wind corrected inducing factor, but not the analysis of lateral wind on the specific impact of wind turbine blades. Yang Jun [8] used relative motion velocity decomposition method, the qualitative analysis of the impact of side wind turbine. Liu Xiong[9] considered the changes of parameters of wind turbine yaw wind conditions influence on the calculation model, the theoretical calculation model is improved. Ma Xinxin[10] studied the effect of the change of wind speed of wind turbine loads on blade coordinates. Qin Datong[11] of variable wind speed of wind turbine control system, the control system of wind turbine is studied to improve the. Endusa Billy Muhando[12] studied the effects of wind on the specific control elements of wind power generation system. In this paper, the blade and the blade element coordinate system coordinates of the velocity vector decomposition and analysis of dynamic model of forward and lateral wind condition in wind turbine device gas effect on the axial velocity into account, analysis of the impact of the additional amplitude of wind turbine blade after the difference.

\section{The Decomposition of Wind}

\section{Mobile Device in the Wind}

Shrink tube, central tube and the bottom link as a whole, the wind turbine and the guide rail is fixed on the ground, there is a gap shrinkage pile, plate .plate can move along the guide rail ,range of wind pressure by changing can move along the track, as shown in figure 1.The range of wind speed in wind mobile device can increase the device using the wind turbine, axial velocity fluctuation[13]. 


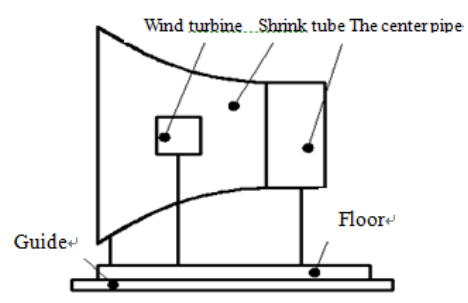

Fig.1 The wind collecting device schematic

\section{The Vector Decomposition of Wind}

In the leaf blade coordinates of the wind vector decomposition velocity component, each component changes with time and space. As show in Figure 2, the wind speed $v$, with angle and spindle $\beta$. The wind speed $v$ can be decomposed into two components, the wind speed $v_{1}$ and direction component of the spindle, known as spindle speed, another component of the wind $v_{2}$ wheel plane flow. The leaf length at $r$ point as the origin, blade length for the $y$ axis, $z$ axis, and $x$ axis $y$ axis and $z$ axis and the axis of the right-handed Cartesian coordinate, the coordinate system is established.

The wind wheel plane leaves to cycle $\varpi$ around the spindle rotation angular velocity, wind wheel plane around the main flow, according to the relative motion of wind speed and wind wheel plane coordinate system, the wind $v_{2}$ in the plane angular frequency around the wind wheel for a week. Surface wind can be decomposed into the coordinate system of $x$ axis and $y$ axis, as shown in Figure 3. As time zero starting point wind and leaves the same direction, then:

$$
\left\{\begin{array}{c}
\vec{v}=\overrightarrow{v_{1}}+\overrightarrow{v_{2}} \\
\overrightarrow{v_{2}=v_{x}+\vec{v}_{y}=}=v_{2} \sin w t \vec{i}+v_{2} \cos w t \vec{j} \\
\vec{v}=\overrightarrow{v_{1}}+\overrightarrow{v_{x}}+\overrightarrow{v_{y}}
\end{array}\right.
$$

Based on equation (1), we can obtain that

$$
\vec{v}=v_{2} \sin w t \vec{i}+v_{2} \cos w t \vec{j}+v \cos \beta \vec{k}
$$

So, $\vec{v}=v \sin \beta \sin w t \vec{i}+v \sin \beta \cos w t \vec{j}+v \cos \beta \vec{k}$

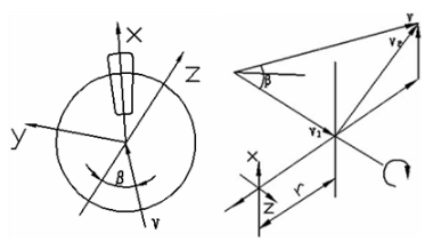

Fig. 2 The blade coordinate velocity diagram

Due to the influence of the axial velocity and nozzle $v_{1}$ cross section area section area increasing , the wind collecting device of wind turbine location section wind speed $v_{N}[13]$ :

$$
v_{1 N}=\frac{S_{1} v_{1}-\frac{1}{2} \mu v_{1} d l_{x}}{\frac{1}{2} \mu d l_{x}+S_{N}}
$$


Among them, $v_{1 N}$ is the axial wind speed position, $f$ is side seam area, $d$ is the width of the gap, $l_{x}$ is wind turbine from the entrance section length, $\mu$ is coefficient of discharge gap, $s_{1}$ is shrink tube entrance area, $s_{1 N}$ is the cross-sectional area of wind turbine location.

When the angle of the wind wheel is $\theta, x$ axis does not change, the $y-z$ plane rotate $\theta$ around the $x$ axis, the components of $v_{y}$ and $v_{1 N}$ respectively in the leaf blade span wise sections and the decomposition of $v_{z 2}$ and $v_{y 2}$; the axial component of the tangential component of $v_{z 2}, v_{x 2}$ and span wise component $v_{y 2}$ into blade element coordinate system. When both $\theta$ and $\beta$ angle side angle, the blade coordinate each component into the wind:

$$
\left\{\begin{array}{c}
v_{x 2}=v \sin \beta \sin w t \\
v_{z 2}=v \sin \beta \cos w t \sin \theta+v_{1 N} \cos \theta \\
v_{y 2}=v \sin \beta \cos w t \cos \theta-v_{1 N} \sin \theta
\end{array}\right.
$$

So, $\vec{v}=\vec{v}_{\mathrm{x} 2}+\vec{v}_{z 2}+\vec{v}_{y 2}$

Among them, $v_{x 2}(\beta, t)$ is the tangential component, $v_{z 2}(\beta, t)$ is the axial component, $v_{z 2}(\beta, t)$ is the direction along the blade span wise component.

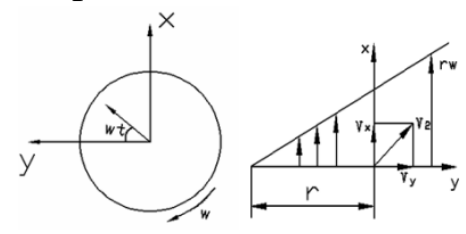

Fig.3 The wind wheel surface wind decomposition

From the above equation, when to flow when the wind is on the wheel, which is equal to 0 , the wind speed above amount does not have the characteristics of temporal and spatial variation. It is because of the presence of unilateral angle, it produced a tangential velocity, and with time since law to change, with a large axial wind speed variation characteristic in the time domain showing changes in accordance with the law of cosine.

\section{Aerodynamic Characteristics}

\section{Forward Aerodynamic Characteristics of Wind Turbine Wind Conditions}

Forward wind represented by $v_{1}$, due to contraction of the tube allows air flow acceleration, set forward wind at the location of wind turbines in the wind unit $v_{1 N} \cdot \theta$ Inclination of the wind wheel, axial wind speed becomes $v_{1 N} \cos \theta, \omega$ speed wind turbine, momentum-based blade element theory and coherent piece wheel, consider wake rotating impeller, $a_{1}$ is the axial induction factor; $a_{2}$ is the hoop or circumferential inducible factor; vasopressin-sectional axial velocity $U_{\mathrm{z}}$ and circumferential velocity $U_{x}$.

$$
U_{\mathrm{x}}=r \omega\left(1+a_{2}\right), \quad U_{\mathrm{z}}=v_{1 N} \cos \theta\left(1-a_{1}\right)
$$

The inflow angle1 and speed $\mathrm{U}$ of vasopressin section

$$
U=\sqrt{U_{x}^{2}+U_{z}^{2}}=\sqrt{v_{1 N}^{2}\left(1-a_{1}\right)^{2} \cos ^{2} \theta+r^{2} \omega^{2}\left(1+a_{2}\right)^{2}} \arctan \frac{U_{z}}{U_{x}}=\delta+\lambda
$$


$\phi$ is the inflow angle; $\delta$ is the angles of attack; $\lambda$ is the blade angle and installation angle of twist . $a_{1}$ and $a_{2}$ after considering loss tip and hub loss correction are:

$$
a_{1}=\frac{B c\left(C_{x} \sin \phi+C_{y} \cos \phi\right)}{8 \pi F r \sin ^{2} \phi+B c\left(C_{x} \sin \phi+C_{y} \cos \phi\right)} \quad a_{2}=\frac{B c\left(C_{y} \sin \phi-C_{x} \cos \phi\right)}{8 \pi F r \sin ^{2} \phi-B c\left(C_{y} \sin \phi-C_{x} \cos \phi\right)}
$$

Among them, $C_{x}$ is the lift coefficient; $C_{y}$ is the drag coefficient; B- is the leaf number; $c$ is the length at radius $\mathrm{r}$ chord; $\mathrm{F}$ is the total loss coefficient; $F=F_{h} F_{t}, F_{h}$ and $F_{t}$ are respectively the hub and tip loss coefficient of loss coefficient, $r_{h u b}$ is the radius of the hub.

$$
F_{h}=\frac{1}{\pi} 2 \operatorname{arc}\left[\exp \left(\frac{B(R-r)}{2 R \sin \phi}\right)\right] \quad F_{t}=\frac{1}{\pi} 2 \operatorname{arc}\left[\exp \left(\frac{B\left(r-r_{h u b}\right)}{2 r_{h u b} \sin \phi}\right)\right]
$$

Given the initial offspring into inducible factor calculated, and then find the normal force coefficient of tangential force coefficient, you can calculate the force on the blade $d T$.

$$
d T=\rho U^{2} B c C_{n} d r / 2
$$

$\rho$ refers to the density of the air; $d r$ is tiny radial length vasopressin; $C_{n}$ is normal force coefficient, $C_{n}=C_{x} \sin \phi+C_{y} \cos \phi C_{t}$ is the tangential force coefficient, $C_{t}=C_{y} \sin \phi-C_{x} \cos \phi$

\section{Calculation of Aerodynamic Force Crosswind Conditions}

In the case of wind, you need to consider the additional wind wheel wind plane, $v$ wind speed, wind speed is $v_{1}$, the spindle device in wind turbine location is indicated by $v_{1 N}$, surface wind with $v_{2}$ said, after the decomposition velocity component to show mainly in the blade surface flow, flow and the contact surface friction, as the effect of surface roughness on the rate of change of the tangential velocity component; the wind wheel wind speed and relative speed are superimposed effect, produce the periodic disturbance, the length of $r$ blade cutting speed to the vector superposition:

$$
V_{t}=r \omega+v_{2} \sin \omega t=r \omega+v \sin \beta \sin \omega t
$$

The relative speed of wind wheel:

$$
\Omega=\frac{V_{t}}{r}=\omega+\frac{v_{2} \sin \omega t}{r}=\omega+\frac{v \sin \beta \sin \omega t}{r}
$$

The axial velocity component:

$$
V_{z}=v_{1 N} \cos \theta+v_{2} \sin \theta \cos \omega t=v_{1 N} \cos \theta+v \sin \beta \sin \theta \cos \omega t
$$

The flow through the blade tangential velocity and axial velocity respectively:

$$
U_{\mathrm{x} 2}=r \Omega\left(1+a_{2}\right)=U_{x}+v \sin \beta \sin w t, \quad U_{z 2}=V_{z}\left(1-a_{1}\right)=U_{a}+v_{2}\left(1-a_{1}\right) \sin \theta \cos \omega t
$$

Wind blade section and flow angle:

$$
U_{2}=\sqrt{U_{x 2}^{2}+U_{z 2}^{2}}, \phi_{2}=\delta_{2}+\varepsilon+\lambda=\phi+\Delta \delta
$$

Because the plane crosswind induced flow, the tangential velocity and the axial velocity with additional items. Seen from the type 13, increased as the length increases, the changes of sine and 
cosine law in time domain. To increase the inflow velocity and angle of attack changes, so that the lift and drag coefficient of the airfoil is changing. The nonlinear $\Delta d T$ is increased, the torque and thrust on the leaf element is expressed as:

$$
d T_{2}=\rho U_{2}^{2} B c C_{n} d r / 2=d T+\Delta d T
$$

\section{The Numerical Calculation}

\section{The Process of Solving Speed Induction Factor}

For each section of the blade torque and thrust, speed is the key to inducing factors, derived $a_{1}$ and $a_{2}$, the iterative procedure is as follows:

1) For the initial $a_{1}$ and $a_{2}$ assignment;

2) Use of type (6) to calculate the inflow angle $\phi$;

3) According to equation (6) section for calculation of angle of attack;

4) According to the data of airfoil lift and find out drag coefficient and angle of attack;

5) According to equation (8) calculate the tip loss and hub loss coefficient;

6) Calculate the value of $a_{1}$ and $a_{2}$;

Return to step 2 iterates until coefficient convergence.

\section{Analysis of the Results}

NACA632[14] FD2-300 parameters of wind turbine airfoil as a reference, as shown in Table 1, the spindle speed is $12 \mathrm{~m} / \mathrm{s}$, surface wind speed of $5 \mathrm{~m} / \mathrm{s}$, by using the method of numerical calculation. In order to avoid the huge computational considering time-varying wind speed, considering only the determined at constant velocity, angle condition, influence of cross wind on aerodynamic performance of wind turbine. This simplification can avoid other uncertainty factors on the analysis results; can be obtained by the single factor affecting wind on aerodynamic performance.

Tab. 1 The wind turbine operation parameters

\begin{tabular}{|c|c|}
\hline The wind wheel diameter $(\mathrm{m})$ & 2 \\
\hline Blade number & 3 \\
\hline The wind speed $(\mathrm{m} / \mathrm{s})$ & 13 \\
\hline${\text { The pitch angle }\left(^{\circ}\right)}^{\circ}$ & $9^{\circ}$ \\
\hline The reference height $(\mathrm{m})$ & $2 \mathrm{~m}$ \\
\hline The rotating speed of the wind wheel $(\mathrm{r} / \mathrm{min})$ & 400 \\
\hline Airfoil series & NACA632 \\
\hline${\text { The density of air }\left(\mathrm{kg} / \mathrm{m}^{3}\right)}^{\circ}$ & 1.225 \\
\hline Wind deflection angle $\left(^{\circ}\right)$ & $22.6^{\circ}$ \\
\hline The wind wheel angle $\left(^{\circ}\right)$ & $0^{\circ}$ \\
\hline
\end{tabular}

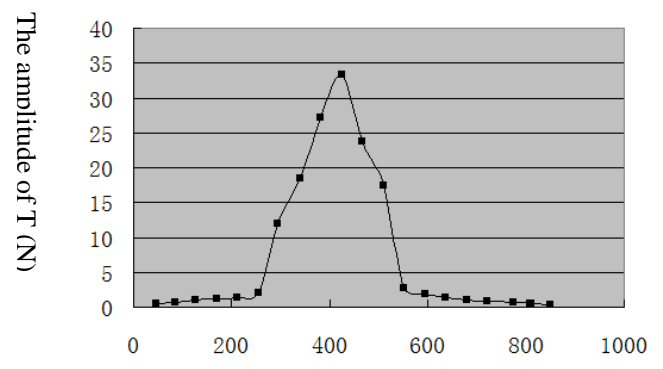

From the center of the wheel hub length (m)

Fig. 5 The diagram of $\Delta T$ Thrust amplitude changes with the length of the blade 
As can be seen from Figure 5, the axial thrust and torque increases suddenly a lot in the radius length 250-450mm regional amplitude analysis, mainly because of surface wind speed air flow pulsation frequency is the blade with the impact of wind turbine main shaft at the same time, also will be affected by the impact of the same frequency torque.

\section{Conclusion}

Crosswind velocity decomposition, the impact of side wind velocity changes reflected in the blade section of axial velocity and tangential velocity with sine, cosine changes, and changes of the axial velocity law. Additional specific solving crosswind induced display it can produce periodic local impact is larger in the middle part of the leaf, is the main cause of wind turbine blade flapping tremor and, to provide support for the effects of crosswinds on wind turbine.

\section{Acknowledgment}

This paper supported by the Natural Science Foundation of Zhejiang Province, China(LY12E09001); the Xinmiao talent foundation of Zhejiang Province (2014R405031).

\section{Reference}

[1]Bo Liu, Zhihao Zhang, Shanfeng He. A preliminary study on wind factors leading to the vibration of wind turbine [J]. Journal of solar energy, 1987, 8( 3): 228-232.

[2] Zhihao Jin, Xuanhua Fan, Pu Chen. The blade of wind turbine wind induced vibration research [J]. Journal of Nanjing University of Aeronautics \& Astronautics,2011, 43(5): 677-681.

[3] J.Gordon Leishman. Challenges in Modelling the Unsteady Aerodynamics of Wind Turbines[J]. WIND ENERGY, 2012, 5(4): 85-132.

[4] Lei Liu, Kechong Shi, Ke Yang. Effect of wind shear loads of wind turbine [J]. Journal of Engineering Thermophysics, 2010, 31(10): 1667-1670.

[5] Huachao Jiao, Wenlei Sun. Characteristic analysis of rotating in 3D aerodynamic blade for large wind turbine [J]. Mechanical design and manufacturing,2010, (6): 98-100.

[6] Liming Zhen, Tong Jiang. Analysis of large horizontal axis wind turbine state [J]. Mechanical and electrical engineering, 998, (2): 537-542.

[7] Yan Wu. Wind turbine blade unsteady aerodynamic characteristics calculation [D]. Nanjing University of Aeronautics and Astronautics, 2005.

[8] Jun Yang, Datong Qin. Influence of side wind on the wind turbine operation [J]. Journal of solar energy, 2011, 32(4): 537-542.

[9] Xiong Liu, Yan Chen, Zhiqaun Ye. Horizontal axis wind turbine aerodynamic performance dispersion model [J]. Journal of solar energy, 2005, 26(6): 792-800.

[10] Xinxin Ma, Jin Chen,Shiliu Chen. Wind turbine blade load distribution under the three dimensional turbulent flow [J]. Chinese Journal of Mechanical Engineering, 2012, 48(7): 1-8.

[11] Datong Qin, Wei Long, Haibo Zhou. Dynamic characteristics of a variable speed wind power operation control of traditional [J]. Chinese Journal of Mechanical Engineering, 2012, 48(7): 1-8.

[12] Endusa Billy Muhando, Tomonobu Senjyu. Augmented LQG controller for enhancement of online dynamic performance for WTG system[J]. Renewable energy, 2008 ,23 (8): 1942-1952.

[13] Hongbin Li, Guofu Li, Xiaozhen Huang. Performance analysis of a mobile device in the wind [J]. Modern scientific instruments, 2014,(1).

[14] Geng Tie, Wenjun Qi. Numerical simulation of horizontal axis wind turbine blade airfoil [J]. Fluid machinery, 2013, 41(3): 29-33. 